\title{
Interaction with neutrophils promotes gastric cancer cell migration and invasion by inducing epithelial-mesenchymal transition
}

\author{
WEN ZHANG $^{1 *}$, JIANMEI GU ${ }^{2 *}$, JINGYAN CHEN $^{1}$, PENG ZHANG $^{1}$, \\ RUNBI JI ${ }^{1}$, HUI QIAN ${ }^{1}$, WENRONG XU ${ }^{1}$ and XU ZHANG ${ }^{1}$ \\ ${ }^{1}$ Jiangsu Key Laboratory of Medical Science and Laboratory Medicine, School of Medicine, \\ Jiangsu University, Zhenjiang, Jiangsu 212013; ${ }^{2}$ Department of Clinical Laboratory, \\ Nantong Tumor Hospital, Nantong, Jiangsu 226361, P.R. China
}

Received March 25, 2017; Accepted August 11, 2017

DOI: 10.3892/or.2017.5942

\begin{abstract}
Emerging evidence has revealed that neutrophils have phenotypic and functional plasticity. Neutrophils could be polarized towards a pro-tumor phenotype by tumor-derived factors. In the present study, we investigated the role of the interaction with neutrophils on the functions of gastric cancer cells in vitro. Human promyelocytic leukemia HL-60 cells were induced to differentiate into neutrophil-like cells (HL-60N) using dimethyl sulfoxide (DMSO). Human gastric cancer cells were co-cultured with HL-60N cells or treated with the conditioned medium (CM) of cancer-activated HL-60N cells. The migration and invasion of gastric cancer cells were significantly enhanced while their proliferation was minimally altered. The expression of pro-inflammatory factors including IL-6, IL-8, IL-1 $\beta$, and TNF $\alpha$ was significantly increased in cancer-activated HL-60N cells, which induced the activation of the ERK pathway and epithelial-mesenchymal transition (EMT) in gastric cancer cells. Blocking the ERK pathway activation reversed the promoting effects of cancer-activated HL-60N cells on gastric cancer cell migration and invasion. In addition, mouse gastric cancer cell derived CM could also increase the expression of pro-inflammatory factors in mouse bone marrow neutrophils, which in turn enhanced the migration and invasion of mouse gastric cancer cells. Collectively, our findings revealed that the interaction with neutrophils promoted gastric cancer cell migration and invasion through the activation of the ERK pathway
\end{abstract}

Correspondence to: Dr Xu Zhang, Jiangsu Key Laboratory of Medical Science and Laboratory Medicine, School of Medicine, Jiangsu University, 301 Xuefu Road, Zhenjiang, Jiangsu 212013, P.R. China

E-mail: xuzhang@ujs.edu.cn

${ }^{*}$ Contributed equally

Key words: neutrophils, EMT, metastasis, microenvironment, gastric cancer and the induction of EMT, indicating that neutrophils may play an important role in gastric cancer metastasis. Therefore, targeting neutrophil-cancer cell interaction may provide a new strategy for the treatment of gastric cancer.

\section{Introduction}

Gastric cancer is the fourth most common cancer and the second leading cause of cancer-related deaths worldwide. Almost half of the patients present with advanced disease at diagnosis and the 5-year survival rate is $<30 \%$ (1). The inflammatory microenvironment has been revealed to be tightly associated with gastric cancer development and progression. Multiple types of cells exist in the gastric cancer microenvironment, including fibroblasts, macrophages, regulatory T cells, and myeloid-derived suppressor cells, which have been demonstrated to play important roles in gastric cancer initiation, growth and metastasis (2). Recent studies have revealed that neutrophils are a predominant component of the tumor microenvironment and that these cells are critically involved in cancer development and progression $(3,4)$. Neutrophils have been reported to promote gastric cancer growth and progression by stimulating angiogenesis and suppressing anti-tumor T-cell responses $(5,6)$.

Increasing evidence has revealed that both human and mouse neutrophils could be polarized towards a pro-tumor $\mathrm{N} 2$ phenotype by tumor-derived factors (7). In a mouse gastrointestinal cancer liver metastasis model, the polarized neutrophils produced substantially higher levels of FGF2, a pro-angiogenic molecule, to support vessel formation and liver metastatic growth (8). In mouse breast cancer, the IL-17-producing $\gamma \delta \mathrm{T}$ cells induced neutrophils to expand and acquire an $\mathrm{N} 2$ phenotype, suppressing $\mathrm{CD}^{+} \mathrm{T}$ cell activation and promoting lung metastasis (9). In human hepatocellular carcinoma (HCC), neutrophils accumulated at the invading tumor edge and represented the major source of HGF, which promoted HCC metastasis via interaction with c-Met in HCC cells (10). In human germinal center B-cell lymphoma, neutrophils could trigger the activation of the nuclear factor- $\kappa \mathrm{B}(\mathrm{NF}-\kappa \mathrm{B})$ pathway in stromal cells, which 
in turn enhanced the survival of malignant B cells (11). The multifaceted roles of neutrophils in the pathogenesis of cancer revealed that a better understanding of the interplay between neutrophils and tumor cells would provide a new approach for cancer therapy.

In our previous study, we demonstrated that neutrophils could act cooperatively with cancer-associated mesenchymal stem cells (MSC) to promote gastric cancer progression (12). In the present study, we further determined the direct effects of neutrophils on gastric cancer cell proliferation, migration and invasion and explored the underlying molecular mechanism. We demonstrated that the interaction with neutrophils promoted gastric cancer cell migration and invasion through the activation of the ERK pathway and the induction of epithelial-mesenchymal transition (EMT), indicating a critical role of neutrophils in gastric cancer metastasis.

\section{Materials and methods}

Cell culture. Human gastric cancer cell lines (MGC80-3 and HGC-27), human promyelocytic leukemia cell line (HL-60), and mouse gastric cancer cell line (MFC) were purchased from the Institute of Biochemistry and Cell Biology of the Shanghai Institutes for Biological Sciences at the Chinese Academy of Sciences (Shanghai, China). MGC80-3 cells were cultured in high-glucose Dulbecco's modified Eagle's medium (DMEM), supplemented with $10 \%$ fetal bovine serum (FBS; Gibco, Invitrogen Life Technologies, Carlsbad, CA, USA). HGC-27, HL-60, and MFC cells were cultured in RPMI-1640 medium containing 10\% FBS. HL-60 cells were induced to differentiate into neutrophil-like cells (HL-60N) by $1.25 \%$ dimethyl sulfoxide (DMSO; Sigma-Aldrich, St. Louis, MO, USA) for 5 days. All the cells were cultured in a humidified incubator with $5 \% \mathrm{CO}_{2}$ at $37^{\circ} \mathrm{C}$. For the cell co-culture experiment, gastric cancer cells were seeded into plates in the presence or absence of HL-60N cells (1:3 ratio).

Conditioned medium (CM) preparation. MGC80-3, HGC-27 and MFC cells were seeded onto $10-\mathrm{cm}$ plates at a density of $8 \times 10^{5}$ cells/plate for $24 \mathrm{~h}$ in complete culture medium. The cells were then washed with phosphate-buffered saline (PBS) twice and cultured in serum-free medium for another $24 \mathrm{~h}$. The supernatants were collected and the cell debris was removed by centrifugation at $3,000 \mathrm{x} \mathrm{g}$ for $10 \mathrm{~min}$. The CM (designated as GC-CM) was filtered through a $0.22-\mathrm{mm}$ filter and stored at $-80^{\circ} \mathrm{C}$ until use. HL-60N cells were seeded in $25-\mathrm{cm}^{2}$ culture cell flasks at a density of $1 \times 10^{5}$ cells and treated with GC-CM (1:1 ratio, v/v) for $24 \mathrm{~h}$, then washed with PBS twice and cultured in serum-free medium for another $24 \mathrm{~h}$. The CM (designated as HL-60 CM) was collected, filtered and stored at $-80^{\circ} \mathrm{C}$.

Cell viability assay. For the cell co-culture, $3 \times 10^{3} \mathrm{GC}$ cells were seeded into each well of 96 well-plates in the presence or absence of HL-60N cells (1:3 ratio). For the CM treatment, the same density of gastric cancer cells was seeded into each well of 96 well-plates and treated with or without HL-60N CM (1:1 ratio, v/v). At different time-points (1, 2 and 3 days) a methyl thiazolyl tetrazolium (MTT) assay was performed. Twenty microliters of
MTT $(5 \mathrm{mg} / \mathrm{ml})$ was added into each well and the cells were further incubated for $4 \mathrm{~h}$. Then, the media were discarded and $150 \mu \mathrm{l}$ DMSO was added. The plate was shaken at room temperature for $15 \mathrm{~min}$ and the absorbance was read at $490 \mathrm{~nm}$ on an automatic microplate reader (Bio-Tek Instruments, Winooski, VT, USA).

Cell colony formation assay. Following the co-culture with HL-60N cells or treatment with HL-60N CM for $48 \mathrm{~h}$, the cells were cultured in six-well plates at a density of 500 cells/well for 8 days. The medium was changed every two days. At the end of the experiment, the cell colonies were fixed with formaldehyde and stained with crystal violet for $30 \mathrm{~min}$. The number of cell colonies was counted under a microscope.

Transwell migration assay. After the co-culture with HL-60N cells or treatment with HL-60N CM for $48 \mathrm{~h}$, the cells were collected and seeded into the upper chamber $(8 \mu \mathrm{m})$ at a density of $1 \times 10^{5}$ cells/well (Corning Inc., Corning, NY, USA). The lower chamber was filled with $500 \mu \mathrm{l}$ culture medium supplemented with $10 \%$ FBS. Nine hours later, the cells on the upper surface of the membrane were removed with a cotton swab. Then, the lower cells were fixed with formaldehyde and stained with crystal violet for $30 \mathrm{~min}$. The number of migrated cells was counted under a microscope.

Cell invasion assay. The diluted basement Matrigel (BD Biosciences, Franklin Lakes, NJ, USA) was added into each chamber and let to polymerize at $37^{\circ} \mathrm{C}$ for $30 \mathrm{~min}$. The cells were seeded into the upper chamber at a density of $2 \times 10^{5}$ cells/well. The lower chamber was filled with $500 \mu \mathrm{l}$ culture medium supplemented with $10 \%$ FBS. The cells were allowed to invade to the lower membrane for $30 \mathrm{~h}$. Subsequently, the cells on the upper surface of the membrane were removed with a cotton swab. The lower cells were then fixed with formaldehyde and stained with crystal violet for $30 \mathrm{~min}$. The number of migrated cells was counted under a microscope.

Quantitative real-time PCR. Total RNA was extracted using TRIzol reagent (Invitrogen, Carlsbad, CA, USA) and reversetranscribed into cDNA using miScript reverse transcription kit (Bio-Rad, Hercules, CA, USA). The relative expression of target genes was detected on a Bio-Rad CFX96 quantitative real-time PCR system with the SYBR Green method ( $\beta$-actin served as an internal control). The sequences of the primers are listed in Table I.

Western blot analysis. The cells were washed twice with PBS and lysed with RIPA buffer containing $1 \%$ protease inhibitors. Equal amounts of proteins were separated on $10 \%$ SDS-polyacrylamide gels and transferred onto polyvinylidene fluoride (PVDF) membranes, followed by blocking with $5 \%$ non-fat milk for $1 \mathrm{~h}$. The membranes were incubated with primary antibodies overnight at $4^{\circ} \mathrm{C}$. The following primary antibodies were used: anti-T-ERK (4695S; Cell Signaling Technology, Beverly, MA, USA), anti-p-ERK (4370S; Cell Signaling Technology), anti-E-cadherin (H-108; Santa Cruz Biotechnology, Dallas, TX, USA), anti-Slug (9585S; Cell Signaling Technology), anti-vimentin (5741S; Cell Signaling 
Table I. The sequences of primers for qRT-PCR.

\begin{tabular}{|c|c|}
\hline Gene & Sequence $\left(5^{\prime}-3^{\prime}\right)$ \\
\hline \multirow[t]{2}{*}{$\beta$-actin } & F: 5'-CACGAAACTACCTTCAACTCC-3' \\
\hline & R: 5'-CATACTCCTGCTTGCTGATC-3' \\
\hline \multirow[t]{2}{*}{ E-cadherin } & F: 5'-CGCATTGCCACATACACTCT-3' \\
\hline & R: 5'-TTGGCTGAGGATGGTGTAAG-3' \\
\hline \multirow[t]{2}{*}{ Slug } & F: 5'-CCTGGTTGCTTCAAGGACAC-3' \\
\hline & R: 5'-TCCATGCTCTTGCAGCTCTC-3' \\
\hline \multirow[t]{2}{*}{ Vimentin } & F: 5'-GAGCTGCAGGAGCTGAATG-3' \\
\hline & R: 5'-AGGTCAAGACGTGCCAGAG-3' \\
\hline \multirow[t]{2}{*}{ ZEB1 } & F: 5'-CAGAAGCCAGTGGTCATGAT-3' \\
\hline & R: 5'-GACTGCGTCACATGTCTTTG-3' \\
\hline \multirow[t]{2}{*}{ Sox 2} & F: 5'-ACACCAATCCCATCCACACT-3' \\
\hline & R: 5'-GCAAACTTCCTGCAAAGCTC-3' \\
\hline \multirow[t]{2}{*}{ Oct4 } & F: 5'-TTGAGGCTCTGCAGCTTAG-3' \\
\hline & R: 5'-GCCGGTTACAGAACCACAC-3' \\
\hline \multirow[t]{2}{*}{ Nanog } & F: 5'-CCTGATTCTTCCACCAGTCC-3' \\
\hline & R: 5'-TGCTATTCTTCGGCCAGTTG-3' \\
\hline \multirow[t]{2}{*}{ IL-1 $\beta$} & F: 5'-TACGAATCTCCGACCACCA-3' \\
\hline & R: 5'-GGACCAGACATCACCAAGC-3' \\
\hline \multirow[t]{2}{*}{ IL-6 } & F: 5'-TACATCCTCGACGGCATCTC-3' \\
\hline & R: 5'-AGCTCTGGCTTGTTCCTCAC-3' \\
\hline \multirow[t]{2}{*}{ IL-8 } & F:5'-GCTCTGTGTGAAGGTGCAGTTT-3' \\
\hline & R: 5'-TTCTGTGTTGGCGCAGTGT-3' \\
\hline \multirow[t]{2}{*}{$\mathrm{TNF} \alpha$} & F: 5'-CCTGCCCCAATCCСТTTA-3' \\
\hline & R: 5'-TGGTTGCCAGCACTTCACT-3' \\
\hline \multirow[t]{2}{*}{ IL-1 $\beta$ (mouse) } & F: 5'-AGCTTCAGGCAGGCAGTATC-3' \\
\hline & R: 5'-TCATCTCGGAGCCTGTAGTG-3' \\
\hline \multirow[t]{2}{*}{ IL-6 (mouse) } & F: 5'-AAGTCCGGAGAGGAGACTTC-3' \\
\hline & R: 5'-TGGATGGTCTTGGTCCTTAG-3' \\
\hline \multirow[t]{2}{*}{$\mathrm{TNF} \alpha$ (mouse) } & F: 5'-AACTCCAGGCGGTGCCTATG-3' \\
\hline & R: 5'-TCCAGCTGCTCCTCCACTTG-3' \\
\hline
\end{tabular}

F, forward; R, reverse.

Technology) and anti-GAPDH (MB001; Bioworld Technology, St. Louis Park, MN, USA). After incubation with the secondary antibodies (Bioworld Technology) at $37^{\circ} \mathrm{C}$ for $1 \mathrm{~h}$, the bands were visualized with an ECL chemiluminescent detection system.

Enzyme-linked immunosorbent assay (ELISA). The HL-60N cells were co-cultured with the HGC-27 cells or treated with the supernatants from the HGC-27 cells for $24 \mathrm{~h}$. The CM of the HL-60N cells was collected. The protein levels of IL-1 $\beta$, IL-6, IL-8 and TNF $\alpha$ were assessed using ELISA kits (Bangyi Biotechnology, Shanghai, China), according to the manufacturer's protocol. A volume of $100 \mu \mathrm{l}$ of culture supernatants was added to each well and the absorbance was assessed at $450 \mathrm{~nm}$. ELISA was performed in triplicate.

Cell sorting. Mice (615; 6-8 weeks old) were purchased from the Tianjin Institute of Hematology and Blood Diseases Hospital (Tianjin, China). The bone marrow was collected and filtered through a 200-mesh metal screen and centrifugated at
$800 \mathrm{x} \mathrm{g}$ for $5 \mathrm{~min}$. After the lysis of the red cells, the single cell suspensions were washed once with PBS containing 2\% FBS and incubated with anti-Ly6G-PE and anti-CD11b-PE Cy7 antibodies for $30 \mathrm{~min}$ at $4^{\circ} \mathrm{C}$. The $\mathrm{CD} 11 \mathrm{~b}^{+} \mathrm{Ly} 6 \mathrm{G}^{+}$neutrophils were isolated on a flow cytometric cell sorter (BD FACS Aria II; BD Biosciences). The present study was approved by the Animal Care and Use Committee of Jiangsu University.

Statistical analysis. All the results were expressed as the mean \pm SD. Statistical analyses were performed using Student's t-test with GraphPad Prism version 5.0 software (GraphPad Software, La Jolla, CA, USA). P $<0.05$ was considered to indicate a statistically significant difference.

\section{Results}

Co-culture with HL-60N cells promotes the migration and invasion of gastric cancer cells. After DMSO induction for 5 days, the expression of CD11b was notably increased in the HL-60N cells, revealing that the HL-60 cells were successfully induced into HL-60N cells (Fig. 1A). To examine the potential role of the HL-60N cells in gastric cancer progression, we co-cultured the gastric cancer cells with HL-60N cells for $48 \mathrm{~h}$ and then examined their proliferating, migratory and invasive abilities. The results of the Transwell migration assay and the Matrigel invasion assay revealed that the co-culture with HL-60N cells markedly increased the migratory and invasive abilities of both the MGC80-3 and the HGC-27 cells (Fig. 1B). However, the results of the MTT and colony formation assays revealed that the gastric cancer cells co-cultured with the HL-60N cells had no significant change in their proliferating ability (Fig. 1C and D). Collectively, these results revealed that the co-culture with HL-60N cells promoted the migration and invasion of GC cells while it did not affect their proliferation.

The CM of gastric cancer (GC)-activated HL-60N cells promotes the migration and invasion of gastric cancer cells. To examine whether the HL-60N cells promoted gastric cancer progression through a cell contact-dependent manner, we collected CM from the HL-60N cells after being co-cultured with HGC-27 cells for $24 \mathrm{~h}$. We then treated the HGC-27 cells with the CM for another $24 \mathrm{~h}$. The metastatic potential of the HGC-27 cells was determined using Transwell invasion assay and Matrigel invasion assay. As shown in Fig. 2A, the CM from the HL-60N cells co-cultured with the HGC-27 cells enhanced the migration and invasion of HGC-27 cells to a greater extent than that from the HL-60N cells alone. The results of the MTT and colony formation assay revealed that there was no significant change in the proliferation of the HGC-27 cells after treatment with the CM from the activated HL-60N cells (Fig. 2C and D). Similar results were also obtained for the MGC80-3 cells (Fig. 2B and D). These results revealed that the $\mathrm{HL}-60 \mathrm{~N}$ cells, in response to the stimuli from gastric cancer cells, promoted the migration and invasion of gastric cancer cells through a paracrine mechanism.

GC-activated HL-60N cells express increased levels of proinflammatory factors. We next determined the expression of pro-inflammatory factors including the IL-6, IL-8, IL-1 $\beta$ and TNF $\alpha$ genes in HL-60N cells using qRT-PCR. We found 

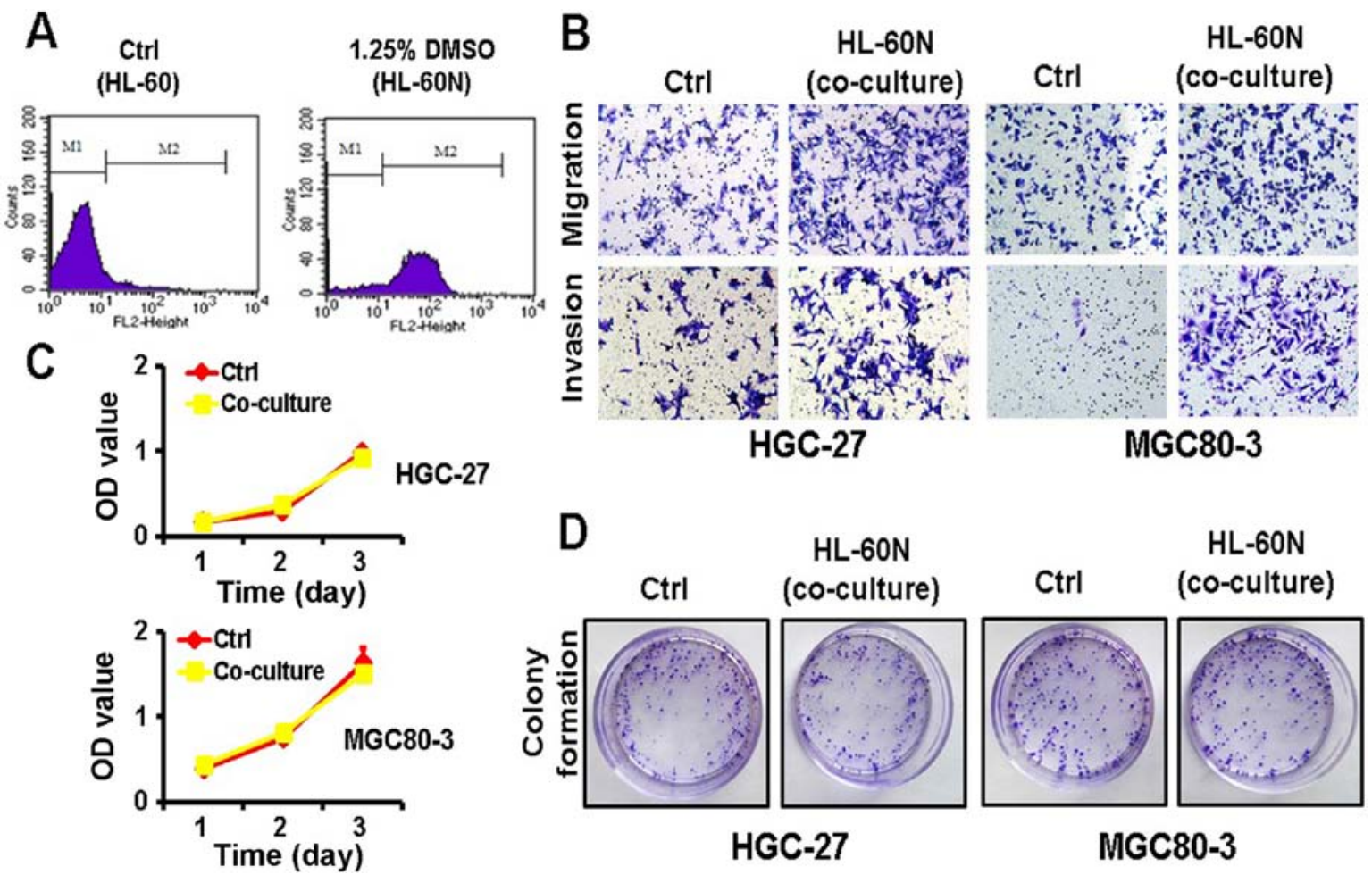

Figure 1. Co-culture with HL-60N cells promotes the migration and invasion abilities of gastric cancer cells. (A) Human HL-60 cells were induced to differentiate into HL- $60 \mathrm{~N}$ by $1.25 \%$ DMSO. The expression of CD11b was determined using flow cytometry. (B) The HL-60N cells were co-cultured with HGC-27 or MGC80-3 cells for $24 \mathrm{~h}$. The migration and invasion of HGC-27 and MGC80-3 cells were determined using Transwell migration assay and Matrigel invasion assay. (C) Cell counting assays for the growth of HGC-27 and MGC80-3 cells. (D) The proliferation of HGC-27 and MGC80-3 cells was determined using cell colony formation assays. HL-60N, neutrophil-like cells; DMSO, dimethyl sulfoxide.
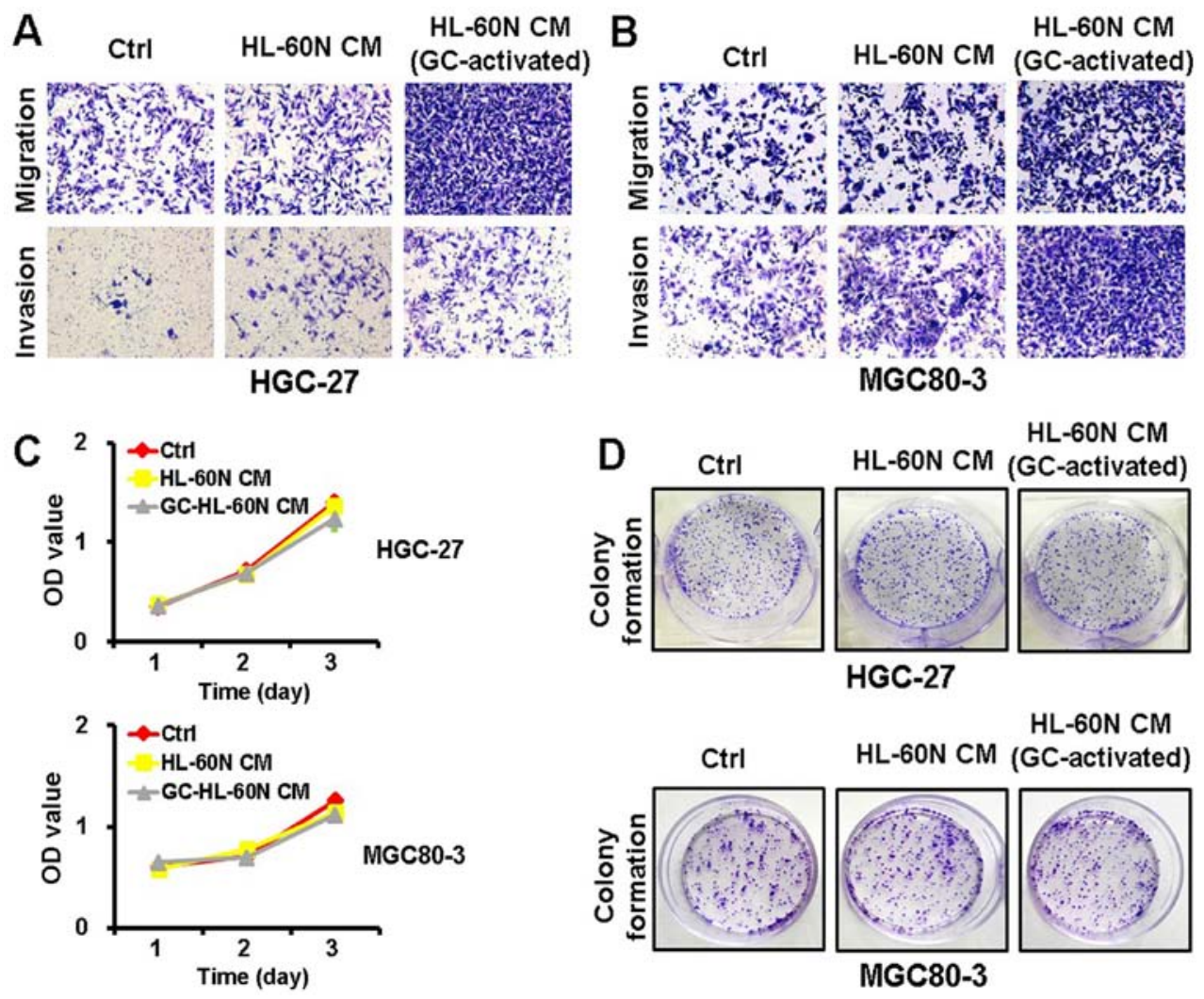

Figure 2. The CM of GC-activated HL-60N cells promotes the migration and invasion of gastric cancer cells. HL-60N cells were treated with the culture supernatants from gastric cancer cells for $24 \mathrm{~h}$. The CM of GC-activated HL-60N cells was collected and used to treat gastric cancer cells as indicated. (A and B) The migration and invasion of HGC-27 cells and MGC80-3 cells were determined using Transwell migration assay and Matrigel invasion assay. (C) Cell counting assays for the growth of HGC-27 and MGC80-3 cells. (D) The proliferation of HGC-27 and MGC80-3 cells was determined using cell colony formation assays. CM, conditioned medium; GC, gastric cancer; HL-60N, neutrophil-like cells. 
A (co-culture with HGC-27 cells)
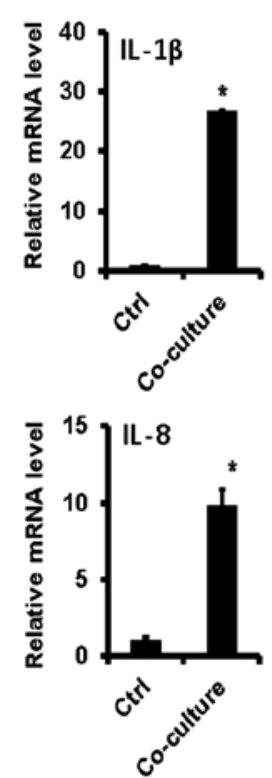
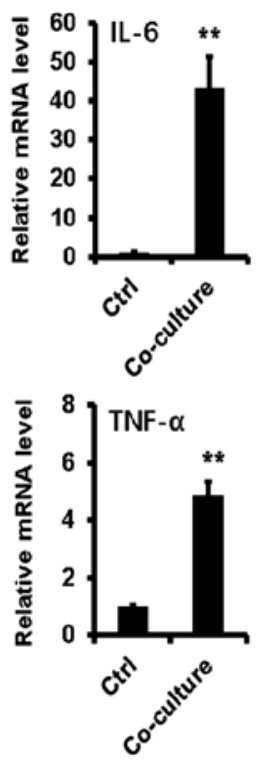

B

$\mathrm{HL}-60 \mathrm{~N}$
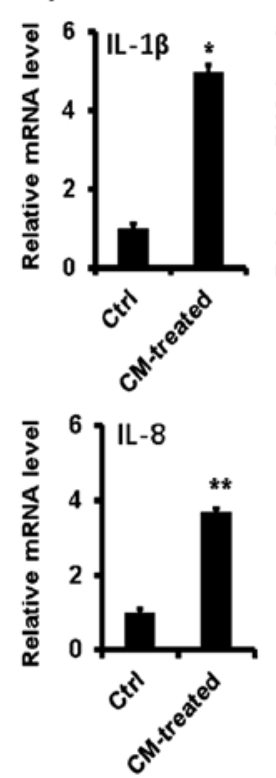
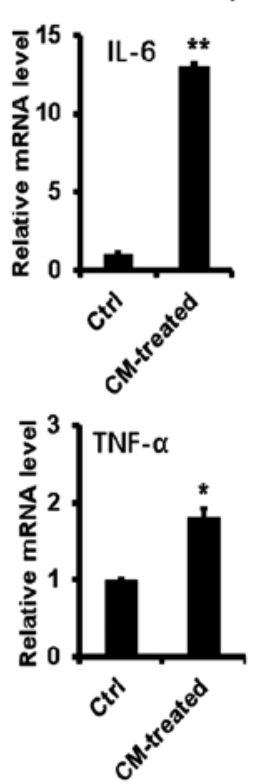

C
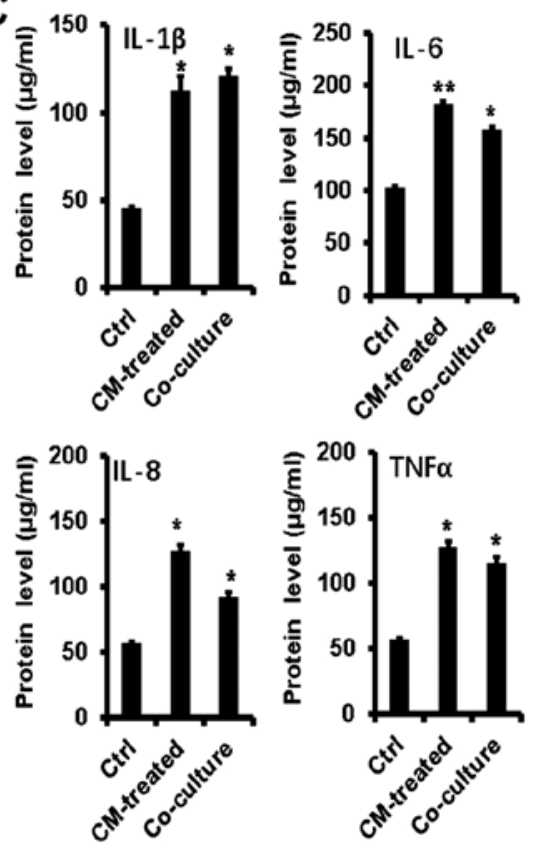

Figure 3. GC-activated HL-60N cells display increased expression of pro-inflammatory factors. (A) The HL-60N cells were co-cultured with HGC-27 cells for $24 \mathrm{~h}$. The expression of the IL-1 $\beta$, IL-6, IL-8, and TNF- $\alpha$ genes in the HL-60N cells was determined using qRT-PCR. (B) The HL-60N cells were treated with the culture supernatants from HGC-27 cells for $24 \mathrm{~h}$. The expression of the IL-1 $\beta$, IL-6, IL-8, and TNF- $\alpha$ genes in the HL-60N cells was determined using qRT-PCR analysis. (C) The expression of the IL-1 $\beta$, IL-6, IL-8 and TNF- $\alpha$ proteins in co-cultured or CM-treated HL-60N cells was determined using ELISA. ${ }^{*} \mathrm{P}<0.05 ;{ }^{* *} \mathrm{P}<0.01$. GC, gastric cancer; HL-60N, neutrophil-like cells; CM, conditioned medium; ELISA, enzyme-linked immunosorbent assay.

that the co-culture with gastric cancer cells significantly upregulated the expression of IL-6, IL-8, IL-1 $\beta$ and TNF $\alpha$ in the HL-60N cells (Fig. 3A). In addition, treatment with the $\mathrm{CM}$ of gastric cancer cells also increased the expression of the IL-6, IL-8, IL-1 $\beta$ and TNF $\alpha$ genes in HL-60N cells (Fig. 3B). Moreover, the results of ELISA revealed that either the co-culture with gastric cancer cells or the treatment with CM of gastric cancer cells improved the expression levels of IL-6, IL-8, IL-1 $\beta$ and TNF $\alpha$ in HL-60N cells (Fig. 3C). Collectively, these results revealed that gastric cancer cells induced HL-60N cells to acquire a pro-inflammatory phenotype.

Co-culture with HL-60N cells or treatment with the CM of activated HL-60N cells induces EMT in gastric cancer cells. We then determined the expression EMT-related genes in gastric cancer cells. The results of the qRT-PCR analyses revealed that the co-culture with $\mathrm{HL}-60 \mathrm{~N}$ cells or treatment with the CM of activated HL- $60 \mathrm{~N}$ cells significantly increased the expression of vimentin, ZEB-1, Slug, Sox2, Nanog and Oct4 in HGC-27 cells (Fig. 4A and B), indicating that the interaction with neutrophils promoted gastric cancer cell migration and invasion through the induction of EMT.

Inhibition of the ERK pathway reverses the promoting effects of the HL-60N cells on gastric cancer cell migration and invasion. We further determined the activation of the ERK pathway in HGC-27 cells using western blot analysis. As shown in Fig. 5A, the activity of p-ERK started to increase at 15 min after treatment with the CM of activated HL-60N cells and was maintained at a high level $2 \mathrm{~h}$ after treatment, while the expression of total ERK exhibited no significant change. To further investigate whether the effects of the activated
HL-60N cells on gastric cancer migration and invasion were ERK pathway-dependent, we pretreated the gastric cancer cells with the ERK inhibitor U0126 for $1 \mathrm{~h}$ and then incubated the cells with the CM of activated HL-60N cells. As shown in Fig. 5B, the inhibition of ERK activity with U0126 could reverse the promoting roles of the activated HL-60N cells in gastric cancer cell migration and invasion. In addition, the results of qRT-PCR revealed that the inhibition of ERK activity with U0126 upregulated the expression of E-cadherin while it downregulated that of vimentin, ZEB-1, Slug, Sox2, Nanog and Oct4 in gastric cancer cells (Fig. 5C). Moreover, the results of the western blot analysis revealed that the inhibition of ERK activity with U0126 upregulated the expression of E-cadherin while it downregulated that of vimentin and Slug (Fig. 5D). These results indicated that the interaction with neutrophils promoted gastric cancer cell migration and invasion through the activation of the ERK pathway.

Mouse bone marrow neutrophils activated by mouse gastric cancer cells promote their migration and invasion abilities. To confirm the results of the in vitro experiments, we isolated neutrophils from the bone marrow of 615 mice by cell sorting. Consistent with the observations for the HL-60N cells, the mouse bone marrow neutrophils also exhibited an increased expression of IL-6, IL-1 $\beta$ and TNF $\alpha$ when treated with the $\mathrm{CM}$ of MFC cells (Fig. 6A). In addition, the CM of activated mouse bone marrow neutrophils could induce the activation of the ERK pathway in mouse gastric cancer MFC cells (Fig. 6B). Furthermore, the promoting roles of the activated mouse bone marrow neutrophils on MFC cell migration and invasion were decreased when the ERK pathway was inhibited (Fig. 6C). Collectively, these results revealed that the activated mouse 
A
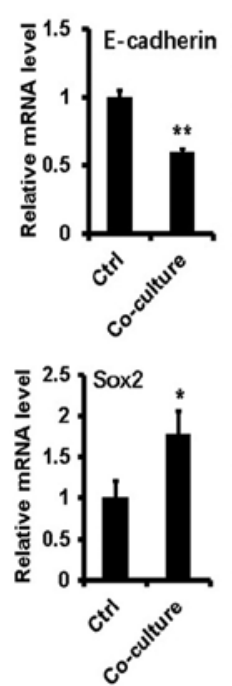

HGC-27

(co-culture with HL-60N cells)
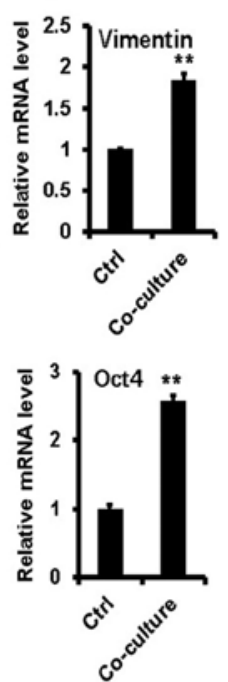
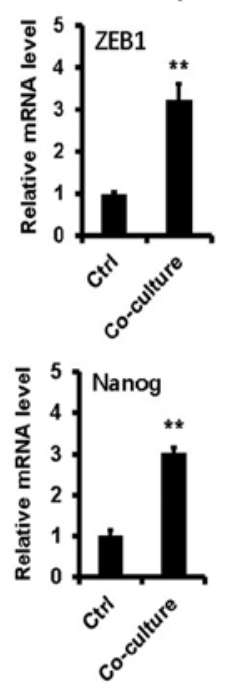

B
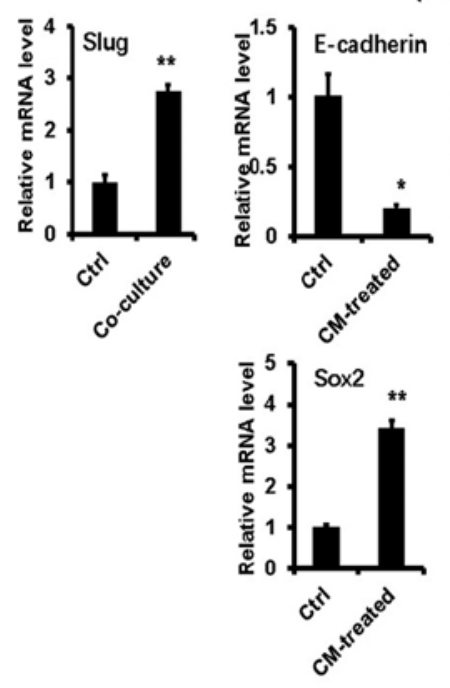

HGC-27

(treated with CM of GC-activated HL-60N cells)
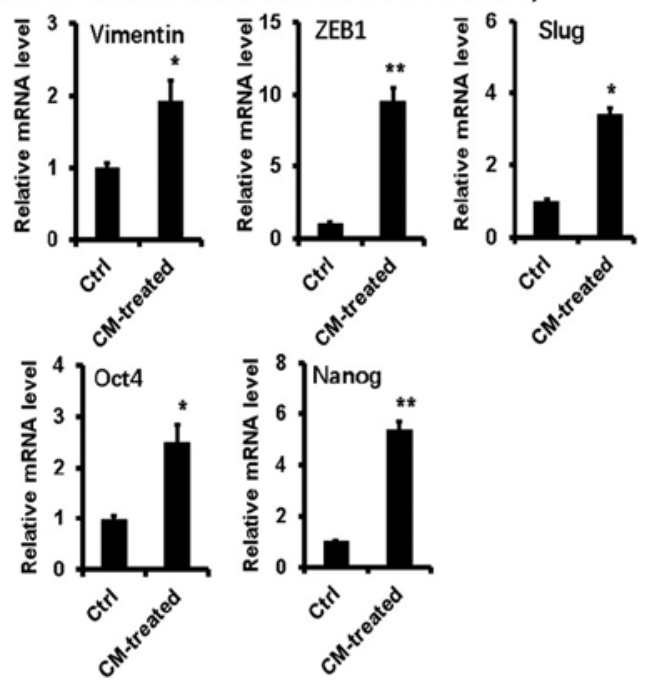

Figure 4. Interaction with HL-60N cells induces EMT in gastric cancer cells. (A) The HGC-27 cells were co-cultured with HL-60N cells for 24 h. The expression of EMT-related genes was determined using qRT-PCR analysis. (B) The MGC80-3 cells were treated with the CM of GC-activated HL-60N cells for $24 \mathrm{~h}$. The expression of EMT-related genes was determined using qRT-PCR analysis. ${ }^{*} \mathrm{P}<0.05 ;{ }^{* *} \mathrm{P}<0.01$. HL-60N, neutrophil-like cells; EMT, epithelialmesenchymal transition; CM, conditioned medium; GC, gastric cancer.

A

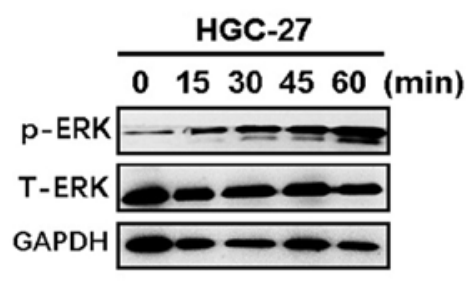

C
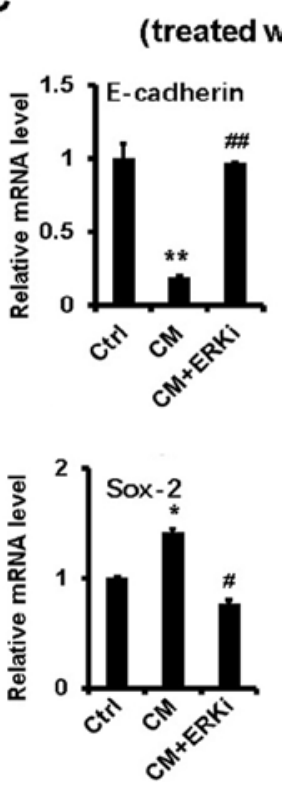

B

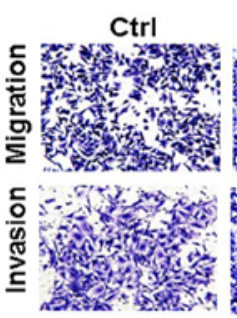

\section{HGC-27}

HGC-27
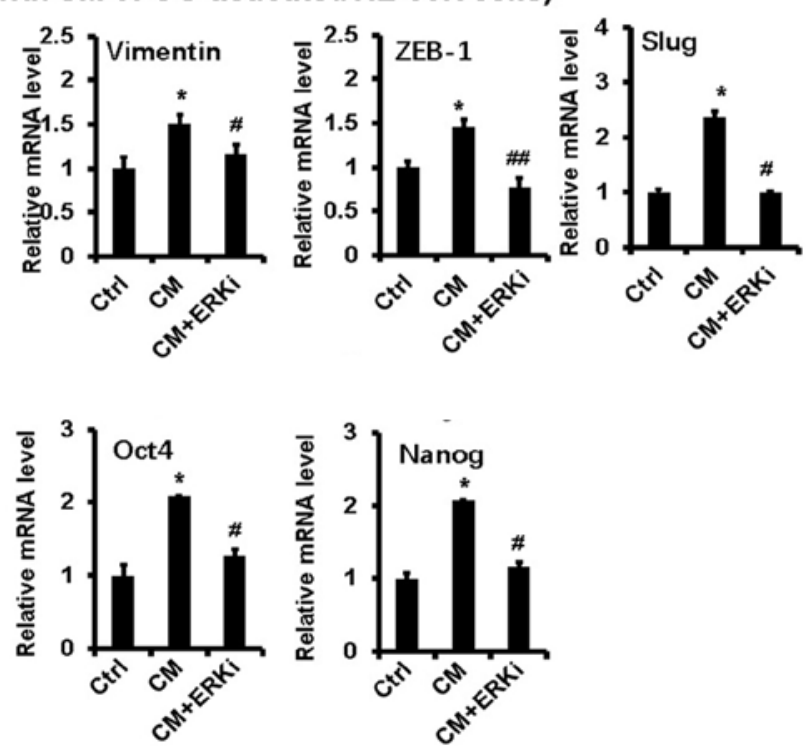

$\underline{\text { HL-60N CM (GC-activated) }}$

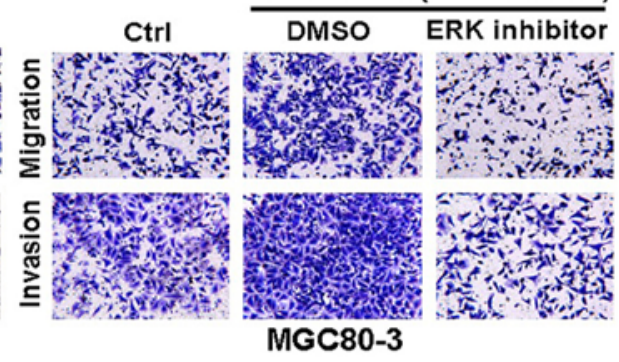

D
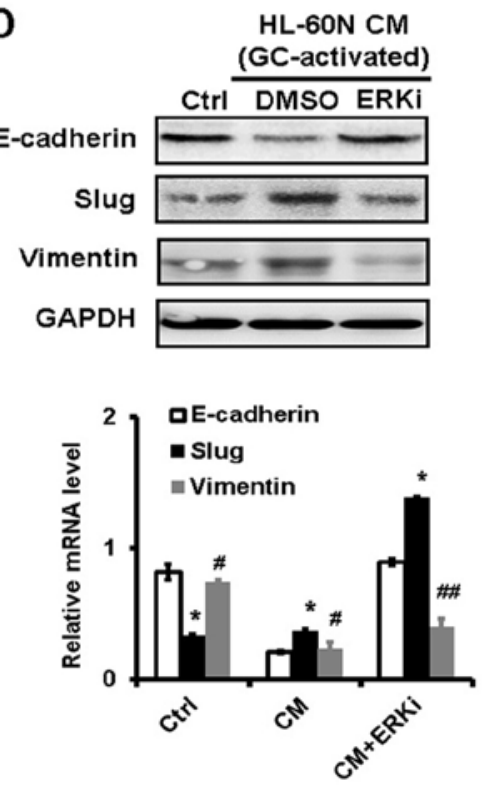

Figure 5. Blocking ERK pathway-activation inhibits the promotion of gastric cancer cell migration and invasion by activated HL-60N cells. (A) HGC-27 cells were treated with the CM of GC-activated HL-60N cells at different time-points as indicated. The expression of p-ERK and T-ERK was determined using western blot analysis. (B) HGC-27 and MGC80-3 cells were treated with the CM of GC-activated HL-60N cells in the presence or absence of the ERK inhibitor U0126 $(10 \mu \mathrm{M})$. The migration and invasion of HGC-27 cells and MGC80-3 cells were determined using Transwell migration assay and Matrigel invasion assay. (C) The expression of EMT-related genes in HGC-27 cells was determined using qRT-PCR analysis. (D) The expression of EMT-related proteins in the HGC-27 cells was determined using western blot analysis. " $\mathrm{P}<0.05$; ${ }^{* *} \mathrm{P}<0.01$, compared to the control group; ${ }^{\#} \mathrm{P}<0.05$; ${ }^{\# \#} \mathrm{P}<0.01$, compared to the $\mathrm{CM}$ group. HL-60N, neutrophil-like cells; CM, conditioned medium; GC, gastric cancer. 


\section{(treated with CM of MFC cells)}
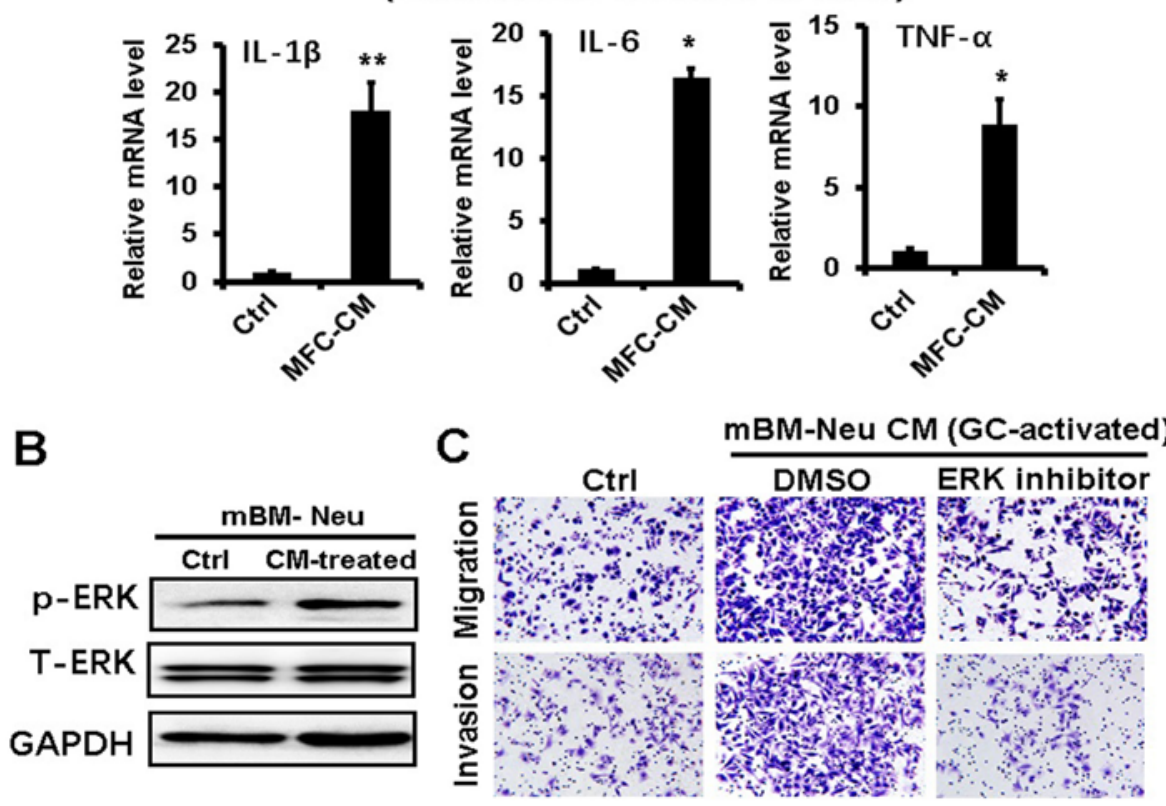

Figure 6. Interaction with mBM-Neu promotes the migration and invasion of mouse gastric cancer cells. (A) Neutrophils were isolated from the bone marrow of 615 mice by flow cytometric cell sorting and treated with the culture supernatants from mouse gastric cancer MFC cells (MFC-CM) for 24 h. The expression of the IL-1 $\beta$, IL-6, and TNF- $\alpha$ genes in mBM-Neu was determined using qRT-PCR. (B) MFC cells were treated with the CM of activated mBM-Neu. The expression of p-ERK and T-ERK was determined using western blot analysis. (C) MFC cells were treated with the CM of activated mBM-Neu in the presence or absence of ERK inhibitor U0126 $(10 \mu \mathrm{M})$. The migration and invasion of MFC cells were determined using Transwell migration assay and Matrigel invasion assay. ${ }^{*} \mathrm{P}<0.05 ;{ }^{* *} \mathrm{P}<0.01$. mBM-Neu, mouse bone marrow-derived neutrophils; $\mathrm{CM}$, conditioned medium.

neutrophils also promoted mouse gastric cancer cell migration and invasion.

\section{Discussion}

In the present study, we demonstrated that human HL-60N cells (induced from the HL-60 cell line using DMSO) and primary neutrophils (isolated from mouse bone marrow) could be activated by gastric cancer to express increased levels of proinflammatory factors, which in turn induced EMT in gastric cancer cells through the activation of the ERK pathway and promoted gastric cancer cell migration and invasion, indicating that the interplay with neutrophils plays a critical role in gastric cancer metastasis.

Neutrophils have been previously revealed to play important roles in tumor metastasis $(3,4)$. Neutrophils could promote tumor metastasis through distinct mechanisms, which include degrading the extracellular matrix, aiding the extravasation of tumor cells and increasing the migratory and invasive abilities of tumor cells. Neutrophils could release proteinases such as catheptin G and elastase to degrade thrombospondin-1 (Tsp-1) and facilitate cancer metastasis (13). Neutrophils released increased levels of oncostatin M (OSM) when stimulated with cancer-derived GM-CSF, which in turn promoted the motility of breast cancer cells (14). Glogauer et al demonstrated that the co-culture with neutrophils increased oral squamous cell carcinoma cell invasion and induced matrix degradation, which was associated with the increase of TNF $\alpha$ and IL-8 in the supernatants from the co-culture system (15). We also found that the co-culture with gastric cancer cells increased the expression of IL-8 and TNF $\alpha$ in activated HL-60N cells and mouse bone marrow neutrophils, which revealed that the induced production of pro-inflammatory factors (e.g., IL-8 and TNF $\alpha$ ) in neutrophils may have profound effects on the promotion of cancer metastasis.

Neutrophils have been reported to induce EMT in tumor cells, which significantly increases the migratory and invasive capacities of tumor cells. Using an oncogenic HRas-induced zebrafish transformation model, Freisinger and Huttenlocher revealed that the expression of EMT-related genes, including Slug and vimentin, was upregulated in the transformed epithelial cells, which was dependent on the presence of neutrophils (16). Hu et al demonstrated that the number of intratumoral neutrophils was negatively associated with E-cadherin expression in human lung cancer tissues (17). Gaida et al revealed that E-cadherin expression was negatively correlated with neutrophil infiltration in the tumor tissues of patients with pancreatic ductal adenocarcinoma (18). Mayer et al suggested that neutrophils induced EMT in human ovarian cancer cells to promote their migration (19). EMT has been demonstrated to critically contribute to gastric cancer development and progression (20). We revealed that the co-culture with neutrophils inhibited the expression of E-cadherin in gastric cancer cells and induced the expression of EMT transcription factors such as ZEB1 and Slug, which in turn enhanced the migratory and invasive capacities of gastric cancer cells. The association between neutrophil infiltration and E-cadherin expression in human gastric cancer tissues warrants further investigation.

The signaling pathway that mediates the promotion of cancer cell migration and invasion by neutrophils varies among various types of cancer. Neutrophils increase HCC cell migration through the activation of the Akt pathway (21). In human lung cancer, neutrophils promoted EMT and 
enhanced the migration of lung cancer cells via the activation of the TGF- $\beta /$ Smad signaling pathway (17). We found that the activated HL-60N cells and mouse bone marrow neutrophils triggered the activation of the ERK pathway in gastric cancer cells. The ERK pathway activation has been previously linked to EMT in gastric cancer (22). Whether other signaling pathways are also involved in the induction of EMT in gastric cancer cells by the activated neutrophils needs to be explored in future studies.

Due to the short life span of human neutrophils, it is difficult to culture these cells in vitro. In order to establish a stable and reliable cell model, we selected the HL-60 cell line in the present study. This cell line could be induced to differentiate into neutrophil-like cells by DMSO and has been ascertained as a model to study the activity and regulation of neutrophils in inflammation and cancer (23). In this study, we found that the HL-60N cells could promote the migration and invasion of gastric cancer cells as that observed in neutrophils from mouse bone marrow, revealing the reliability of using this cell line. However, the limitation of using this cell line is that it may not reflect the real state of human neutrophils. Further studies are warranted to assess the effects of human neutrophils on gastric cancer cell migration and invasion.

In conclusion, our findings revealed that the interaction with neutrophils promoted gastric cancer cell migration and invasion through the induction of EMT. Our data not only added new information to the important roles of neutrophils in cancer metastasis, but also provided a new strategy for the treatment of gastric cancer.

\section{Acknowledgements}

The present study was supported by the National Natural Science Foundation of China (nos. 81672416 and 81201660), the Natural Science Foundation of Jiangsu Province (no. BK20141303), the Key Research and Development Project of Zhenjiang (no. SH2015034), the Starting Foundation for Senior Talents of Jiangsu University (no. 13JDG086), the Qing Lan project of Jiangsu Province, the '333' project of Jiangsu Province and the Foundation for Young Academic Leader of Jiangsu University.

\section{References}

1. Chen W, Zheng R, Baade PD, Zhang S, Zeng H, Bray F, Jemal A, Yu XQ and He J: Cancer statistics in China, 2015. CA Cancer J Clin 66: 115-132, 2016.

2. Ma HY, Liu XZ and Liang CM: Inflammatory microenvironment contributes to epithelial-mesenchymal transition in gastric cancer. World J Gastroenterol 22: 6619-6628, 2016.

3. Zhang X, Zhang W, Yuan X, Fu M, Qian H and Xu W: Neutrophils in cancer development and progression: Roles, mechanisms, and implications (Review). Int J Oncol 49: 857-867, 2016.

4. Coffelt SB, Wellenstein MD and de Visser KE: Neutrophils in cancer: Neutral no more. Nat Rev Cancer 16: 431-446, 2016.

5. Li TJ, Jiang YM, Hu YF, Huang L, Yu J, Zhao LY, Deng HJ, Mou TY, Liu H, Yang Y, et al: Interleukin-17-producing neutrophils link inflammatory stimuli to disease progression by promoting angiogenesis in gastric cancer. Clin Cancer Res 23: $1575-1585,2017$
6. Wang TT, Zhao YL, Peng LS, Chen N, Chen W, Lv YP, Mao FY, Zhang JY, Cheng P, Teng YS, et al: Tumour-activated neutrophils in gastric cancer foster immune suppression and disease progression through GM-CSF-PD-L1 pathway. Gut: Mar 8, 2017 (Epud ahead of print). doi: 10.1136/gutjnl-2016-313075.

7. Powell DR and Huttenlocher A: Neutrophils in the tumor microenvironment. Trends Immunol 37: 41-52, 2016.

8. Gordon-Weeks AN, Lim SY, Yuzhalin AE, Jones K, Markelc B, Kim KJ, Buzzelli JN, Fokas E, Cao Y, Smart S, et al: Neutrophils promote hepatic metastasis growth through fibroblast growth factor 2-dependent angiogenesis in mice. Hepatology 65: 1920-1935, 2017.

9. Coffelt SB, Kersten K, Doornebal CW, Weiden J, Vrijland K, Hau CS, Verstegen NJM, Ciampricotti M, Hawinkels LJAC, Jonkers $\mathbf{J}$ and de Visser KE: IL-17-producing $\gamma \delta \mathrm{T}$ cells and neutrophils conspire to promote breast cancer metastasis Nature 522: 345-348, 2015.

10. He M, Peng A, Huang XZ, Shi DC, Wang JC, Zhao Q, Lin H, Kuang DM, Ke PF and Lao XM: Peritumoral stromal neutrophils are essential for c-Met-elicited metastasis in human hepatocellular carcinoma. OncoImmunology 5: e1219828, 2016.

11. Grégoire M, Guilloton F, Pangault C, Mourcin F, Sok P, Latour M, Amé-Thomas P, Flecher E, Fest T and Tarte K: Neutrophils trigger a NF- $\kappa$ B dependent polarization of tumorsupportive stromal cells in germinal center B-cell lymphomas. Oncotarget 6: 16471-16487, 2015

12. Zhu Q, Zhang X, Zhang L, Li W, Wu H, Yuan X, Mao F, Wang M, Zhu W, Qian H, et al: The IL-6-STAT3 axis mediates a reciprocal crosstalk between cancer-derived mesenchymal stem cells and neutrophils to synergistically prompt gastric cancer progression. Cell Death Dis 5: e1295, 2014.

13. El Rayes T, Catena R, Lee S, Stawowczyk M, Joshi N, Fischbach C, Powell CA, Dannenberg AJ, Altorki NK, Gao D, et al: Lung inflammation promotes metastasis through neutrophil proteasemediated degradation of Tsp-1. Proc Natl Acad Sci USA 112: 16000-16005, 2015.

14. Queen MM, Ryan RE, Holzer RG, Keller-Peck CR and Jorcyk CL: Breast cancer cells stimulate neutrophils to produce oncostatin M: Potential implications for tumor progression. Cancer Res 65: 8896-8904, 2005.

15. Glogauer JE, Sun CX, Bradley G and Magalhaes MA: Neutrophils increase oral squamous cell carcinoma invasion through an invadopodia-dependent pathway. Cancer Immunol Res 3: 12181226,2015

16. Freisinger $\mathrm{CM}$ and Huttenlocher A: Live imaging and gene expression analysis in zebrafish identifies a link between neutrophils and epithelial to mesenchymal transition. PLoS One 9: e112183, 2014.

17. Hu P, Shen M, Zhang P, Zheng C, Pang Z, Zhu L and Du J: Intratumoral neutrophil granulocytes contribute to epithelial-mesenchymal transition in lung adenocarcinoma cells. Tumour Biol 36: 7789-7796, 2015.

18. Gaida MM, Steffen TG, Günther F, Tschaharganeh DF, Felix K, Bergmann F, Schirmacher P and Hänsch GM: Polymorphonuclear neutrophils promote dyshesion of tumor cells and elastase-mediated degradation of E-cadherin in pancreatic tumors. Eur J Immunol 42: 3369-3380, 2012.

19. Mayer C, Darb-Esfahani S, Meyer AS, Hübner K, Rom J, Sohn C, Braicu I, Sehouli J, Hänsch GM and Gaida MM: Neutrophil granulocytes in ovarian cancer - induction of epithelial-to-mesenchymal-transition and tumor cell migration. J Cancer 7: 546-554, 2016.

20. Huang L, Wu RL and Xu AM: Epithelial-mesenchymal transition in gastric cancer. Am J Transl Res 7: 2141-2158, 2015.

21. Wu Y, Zhao Q, Peng C, Sun L, Li XF and Kuang DM: Neutrophils promote motility of cancer cells via a hyaluronan-mediated TLR4/PI3K activation loop. J Pathol 225: 438-447, 2011.

22. Zhou Q, Wang X, Yu Z, Wu X, Chen X, Li J, Zhu Z, Liu B and Su L: Transducin ( $\beta$ )-like $1 \mathrm{X}$-linked receptor 1 promotes gastric cancer progression via the ERK1/2 pathway. Oncogene 36: 1873-1886, 2017.

23. Li XF, Chen DP, Ouyang FZ, Chen MM, Wu Y, Kuang DM and Zheng L: Increased autophagy sustains the survival and pro-tumourigenic effects of neutrophils in human hepatocellular carcinoma. J Hepatol 62: 131-139, 2015. 